\title{
Upper bound for the time derivative of entropy for nonequilibrium stochastic processes
}

\author{
Bidhan Chandra Bag* \\ Department of Chemistry, Visva-Bharati, Santiniketan 731 235, India
}

(October 25, 2018)

\begin{abstract}
We have shown how the intrinsic properties of a noise process can set an upper bound for the time derivative of entropy in a nonequilibrium system. The interplay of dissipation and the properties of noise processes driving the dynamical systems in presence and absence of external forcing, reveals some interesting extremal nature of the upper bound.
\end{abstract}

PACS number(s) : 05.45.-a, 05.70.Ln, 05.20.-y

*e-mail: pcbcb@yahoo.com 


\section{INTRODUCTION}

A consequence of the second law of thermodynamics is that the rate of change of entropy with time for a nonequilibrium stochastic process is always positive. While in traditional classical thermodynamics, the specific nature of a stochastic process is irrelevant, this may play an important role in understanding the connection between the phase space of a dynamical system and the related thermodynamically inspired quantities like entropy production, flux etc. The relationship has recently been explored by a number of authors [1] 13. The object of the present paper is to address a related issue.

In what follows we shall be concerned with the dissipative dynamical systems which are thermodynamically open [14] in the sense that they can be described by classical stochastic processes with the help of the standard Langevin equations. When a dynamical system is driven by a noise process, e. g, color [15] or cross-correlated processes [16, 17], the nature of the noise processes may influence the dynamical system through the appropriate modification of the phase space structure of the overall system. In view of the immediate connection between information entropy and probability distribution function of the phase space variables, it is worthwhile to enquire about the imprints of the nature of noise on entropy. Our specific aim in this paper is to show how the properties of the noise processes can set an upper bound on the rate of entropy change in a nonequlibrium system. By directly extending our earlier treatment on a related problem [1] we have examined some interesting extremal properties of this bound.

The outline of the paper is as follows: In Sec. II we introduce the Fokker-Planck description of a dynamical system driven by two different kinds of stochastic processes (e. g, color and cross-correlated) and an upper bound for the rate of entropy change based on this formulation. We illustrate the result in Section III for the two specific cases. The paper is concluded in Section IV. 


\section{THE FOKKER-PLANCK DESCRIPTION OF NOISE PROCESSES AND UPPER BOUND FOR THE RATE OF ENTROPY CHANGE}

We consider a dynamical system driven by the external Ornstein-Uhlenbeck noise processes. The relevant Langevin equations of motion can be written as

$$
\dot{x}_{i}=F_{i}^{0}\left(\left\{x_{i}\right\}\right)+\eta_{i}, \quad i=1, \cdots, N
$$

where $N$ is the dimension of the phase space. $F_{i}^{0}(x)$ corresponds to the dissipative term as well as the external applied deterministic force, if any. The second term $\eta_{i}$ in Eq.(1) refers to an external, Gaussian, color noise for the i-th component of $x$.

The Fokker-Planck equation corresponding to Langevin Eq.(1) in the extended phase space can be written as [for details, see Ref. [11]]

$$
\frac{\partial \rho(X, t)}{\partial t}=-\sum_{i=1}^{2 N} \frac{\partial}{\partial X_{i}}\left(F_{i} \rho\right)+\sum_{i=1}^{2 N} D_{i} \frac{\partial^{2} \rho}{\partial X_{i}^{2}}
$$

where

$$
X_{i} \begin{cases}=x_{i} & \text { for } i=1, \cdots, N \\ =\eta_{i} & \text { for } i=N+1, \cdots, 2 N\end{cases}
$$

$F_{i}$ and $D_{i}$ are drift and diffusion coefficients, respectively, and have their usual significance as discussed in Ref. [11]. $\rho(X, t)$ is the extended phase space probability distribution function.

As a second example we consider a dynamical system driven by both additive and multiplicative noise processes $\eta_{i}$ and $\zeta_{i}$, respectively. The Langevin equation for this process, in general, can be written as

$$
\dot{X}_{i}=L_{i}\left(\left\{X_{i}\right\}, t\right)+g_{i}\left(X_{i}\right) \zeta_{i}+\eta_{i} \quad i=1, \cdots, N
$$

$L_{i}$ contains the dissipative term as well as the external applied deterministic force, if any. $g_{i}\left(X_{i}\right)$ is the coupling between the system and the multiplicative processes $\zeta_{i}$. $\zeta_{i}$ and $\eta_{i}$ are white, Gaussian noise processes with the following correlation between them;

$$
\left\langle\zeta_{i}(t) \eta_{j}\left(t^{\prime}\right)\right\rangle=\left\langle\zeta_{i}\left(t^{\prime}\right) \eta_{j}(t)\right\rangle=2 \lambda_{i j} \sqrt{D_{i j}^{\prime} \alpha_{i j}} \delta\left(t-t^{\prime}\right) \delta_{i j}
$$


$D_{i j}^{\prime}$ and $\alpha_{i j}$ correspond to the strength of multiplicative and additive noises, respectively and $\lambda$ represents the cross correlation between them with the limit $0 \leq \lambda \leq 1$.

The Fokker-Planck equation corresponding to Langevin Eq.(3) can be written as

$$
\frac{\partial \rho(X, t)}{\partial t}=-\sum_{i=1}^{N} \frac{\partial}{\partial X_{i}}\left(F_{i} \rho\right)+\sum_{i=1}^{N} D_{i} \frac{\partial^{2} \rho}{\partial X_{i}^{2}} .
$$

Again $F_{i}$ and $D_{i}$ are drift and diffusion coefficient, respectively, and have same significance as in the Eqs. (11-13) of Ref. [11].

The Fokker-Planck Eqs.(2) or (5) can be rearranged into the general form of continuity equation

$$
\frac{\partial \rho(X, t)}{\partial t}=-\nabla_{X} \cdot j
$$

where $j$ denotes the current and $\nabla_{X}$ term the phase space divergence. The $i$-th component of $j$ can be written as

$$
j_{i}=F_{i} \rho-D_{i} \frac{\partial \rho}{\partial X_{i}}
$$

Using Eq.(6) we are now in a position to define the upper bound for the rate of evolution of entropy. In the microscopic picture, the Shannon form of the entropy is connected to the probability density function $\rho(X, t)$ as

$$
S=-\int d X \rho(X, t) \ln \rho(X, t) .
$$

The time evolution equation for entropy can then be written as

$$
\frac{d S}{d t}=\int d X \nabla_{X} \cdot j \ln \rho
$$

where Eq.(6) is used.

Integrating Eq.(9) by parts, one obtains

$$
\frac{d S}{d t}=-\int d X \frac{1}{\rho} j . \nabla_{X} \rho,
$$

where we have used following boundary conditions [6] 


$$
\left.j\right|_{\text {boundary }}=0
$$

and

$$
\left.j \ln \rho\right|_{\text {boundary }}=0
$$

In the next step an application of the Schwartz inequality $\left|\int d X g h\right|^{2} \leq \int d X|g|^{2} \int d X|h|^{2}$ to the integral (10) where $g$ and $h$ can be appropriately identified yields an upper bound for the rate of entropy change

$$
\begin{gathered}
\frac{d S}{d t} \leq U_{B}(t) \\
U_{B}(t)=\left(\int d X \frac{j^{2}}{\rho}\right)^{1 / 2} \times\left(\int d X \frac{\left(\nabla_{X} \rho\right)^{2}}{\rho}\right)^{1 / 2}
\end{gathered}
$$

It is important to note that the second integral is same as the trace of Fisher information matrix [8] and this inequality is valid if and only if $j / \sqrt{\rho}$ is not a constant multiple of $\left(\nabla_{X} \rho\right) / \sqrt{\rho}$. To find the explicit time dependence of the upper bound we work out simple examples for each of the noise processes in the next section.

\section{APPLICATIONS}

\section{A. The upper bound for a dynamical system driven by an external color noise}

As a simple illustration, we consider a Langevin equation of motion for a dissipative dynamical system driven by an external, Gaussian Ornstein-Uhlenbeck noise, $\eta_{1}$.

$$
\dot{X}_{1}=-\gamma X_{1}+f_{c}+\eta_{1}
$$

The noise correlation of $\eta_{1}$ is given by

$$
\left\langle\eta_{1}(t) \eta_{1}\left(t^{\prime}\right)\right\rangle=\frac{D^{0}}{\tau} \exp \left(-\frac{\left|t-t^{\prime}\right|}{\tau}\right)
$$

$\gamma$ in Eq.(14) is the dissipative parameter and $f_{c}$ is a constant external applied force term which is used to identify specific interplay between $\gamma$ and $\tau$. 
For the Langevin Eq. (14) the Fokker-Planck Eq.(2) becomes (see Ref.[11])

$$
\frac{\partial \rho}{\partial t}=\gamma \frac{\partial X_{1} \rho}{\partial X_{1}}-f_{c} \frac{\partial \rho}{\partial X_{1}}-X_{2} \frac{\partial \rho}{\partial X_{1}}+\frac{1}{\tau} \frac{\partial X_{2} \rho}{\partial X_{2}}+\frac{D^{0}}{\tau^{2}} \frac{\partial^{2} \rho}{\partial X_{2}^{2}}
$$

where $X_{2}=\eta_{1}$.

We now use the following transformation in the Eq.(16)

$$
U=a X_{1}+X_{2}
$$

where $a$ is a constant to be determined.

Then Eq.(16) reduces into the following one dimensional form:

$$
\frac{\partial \rho(U, t)}{\partial t}=\frac{\partial}{\partial U}(\Gamma U) \rho-F_{u} \frac{\partial \rho}{\partial U}+D_{s} \frac{\partial^{2} \rho}{\partial U^{2}}
$$

where

$$
\Gamma U=\gamma a X_{1}-a X_{2}+\frac{X_{2}}{\tau}, F_{u}=a f_{c} \text { and } D_{s}=\frac{D^{0}}{\tau^{2}}
$$

Here $\Gamma$ is again a constant to be determined. Using Eq.(17) in Eq. (19) and comparing the coefficients of $X_{1}$ and $X_{2}$ we find

$$
\Gamma=\gamma \text { and } a=\frac{1-\gamma \tau}{\tau}
$$

We then search for the Green's function or conditional probability solution for the system at $U$ at time $t$ for the initial condition given by

$$
\rho(U, t=0)=\lim _{\epsilon \rightarrow \infty} \frac{\epsilon}{\pi} \exp \left[-\epsilon\left(U-U^{\prime}\right)^{2}\right]
$$

We now look for a solution of the Eq.(18) of the form

$$
\rho\left(U, t \mid U^{\prime}, 0\right)=\exp [G(t)]
$$

where

$$
G(t)=-\frac{1}{\sigma(t)}(U-\beta(t))^{2}+\ln \nu(t)
$$


We will see that by suitable choice of $\beta(t), \sigma(t), \nu(t)$ we can solve Eq.(18) subject to the initial condition

$$
\rho\left(U, 0 \mid U^{\prime}, 0\right)=\lim _{\epsilon \rightarrow \infty} \frac{\epsilon}{\pi} \exp \left[-\epsilon\left(U-U^{\prime}\right)^{2}\right]
$$

Comparison of this with $(22)$ and $G(0)$ shows that

$$
\sigma(0)=\frac{1}{\epsilon}, \quad \beta(0)=U^{\prime}, \quad \nu(0)=\frac{\epsilon}{\pi}
$$

If we put (22) in (18) and equate the coefficients of equal powers of $U$ we obtain after some algebra the following set of equations

$$
\begin{gathered}
\dot{\sigma(t)}=-2 \Gamma \sigma(t)+4 D_{s} \\
\dot{\beta(t)}=-\beta(t)+F_{u} \\
\frac{1}{\nu(t)} \dot{\nu(t)}=-\frac{1}{2 \sigma(t)} \dot{\sigma(t)}
\end{gathered}
$$

The relevant solutions of $\sigma(t)$ and $\beta(t)$ for the present problem which satisfy the initial conditions as stated earlier are given by

$$
\sigma(t)=\frac{2 D_{s}}{\Gamma}(1-\exp (-2 \Gamma t))+\sigma(0) \exp (-2 \Gamma t)
$$

and

$$
\beta(t)=\frac{F_{u}}{\Gamma}(1-\exp (-2 \Gamma t))+\beta(0) \exp (-\Gamma t)
$$

Now making use of Eqs. (22), (29) and (30) in (13) we finally obtain explicit time dependence of the upper bound $U_{B}(t)$ for the rate of entropy change as

$$
\frac{d S}{d t} \leq U_{B}(t)
$$

where

$$
U_{B}(t)=\frac{\left(2 \beta^{2} \Gamma^{2} \sigma-4 \beta \Gamma F_{u} \sigma+2 F_{u}^{2} \sigma+\Gamma^{2} \sigma^{2}+4 D_{s}^{2}-4 D_{s} \Gamma \sigma\right)^{1 / / 2}}{\sigma}
$$


We now examine specifically the long time limit, i. e, $t \rightarrow \infty$ of the above result (31). As $t \rightarrow \infty$ Eqs. (29) and (30) reduce to

$$
\sigma(\infty)=\frac{2 D_{s}}{\Gamma} \text { and } \beta(\infty)=\frac{F_{u}}{\Gamma}
$$

It is easy to check that as $t \rightarrow \infty$ the numerator of the right hand side of Eq.(31) vanishes both in presence or absence of $F_{u}$. Therefore we obtain the equation

$$
\frac{d S}{d t}=0
$$

This equality holds since in the long time limit $j=0$ (see Eq. 18). At any other time the time dependence of the upper bound $U_{B}$ for the rate of entropy change is explicitly shown in Fig.1. We choose the initial conditions $\sigma(0)=0, \beta(0)=1.0$ and parameter values $D^{0}=1.0, f_{c}=1.0, \gamma=1.0$ and $\tau=1.0$. Fig. 1 shows that except for an initial short period $U_{B}(t)$ decreases almost exponentially with time. In absence of $f_{c}$ the time dependence of $U_{B}$ follows a similar pattern. In Fig.2(a-b) we plot $U_{B}$ at $t=5$ vs correlation time $\tau$ in absence and presence of the external forcing $f_{c}$. As expected $U_{B}$ increases monotonically with the $\tau$ [in Fig.2(a)] which is a clear signature of the persistence of the nonequilibrium situation in contrast to the case in Fig.2(b) where the interplay of $\tau$ with external forcing $f_{c}$ results in a minimum in $U_{B}$. The result of Fig.2(b) is qualitatively same to that of the Fig.1 of Ref.[11] where only entropy production in the stationary state is considered. In the present context however the upper bound of the sum of entropy production and entropy flux [11] at any arbitrary time is considered. The relation between entropy flux $\left(E_{F}\right)$ and entropy production $\left(E_{P}\right)$ in the long time limit for the present model [11] is

$$
E_{P}=-E_{F}=\frac{(1-\gamma \tau)^{2} f_{c}^{2}}{D^{0}}
$$

Using above equation in Eq.(31) at time $t \rightarrow \infty$ we have

$$
U_{B}=\left[2 \gamma E_{P}+2 \gamma E_{F}\right]^{1 / 2}=0 .
$$

Since near equilibrium $E_{P}$ approaches $-E_{F}$ the upper bound of time derivative of entropy as shown in Fig.2(b) mimics the result of Fig.1 of Ref.[11]. 
In Fig. 3(a-b) we plot the variation of $U_{B}$ (at $\left.t=5.0\right)$ vs dissipative constant $\gamma$ in absence $(3 \mathrm{a})$ and presence $(3 \mathrm{~b})$ of the external force $f_{c}$. While an increase in $\gamma$ facilitates the approach to stationarity as evident from the monotonic decrease of the bound in Fig. 3(a), its effect becomes more interesting when the external $f_{c}$ is switched on (Fig. 3(b)). One observes that the bound passes first through minimum followed by a maximum to settle down at the vanishing level for the large values of dissipation. It is thus apparent that in presence of the external constraint the properties of the noise processes and the dynamical

characteristics of the system play an important part for the upper bound for the rate of entropy change.

\section{B. The upper bound in a cross-correlated noise driven system}

We now turn to the second case where a simple dissipative system is driven by both additive and multiplicative noises.

$$
\dot{X}_{1}=-\gamma X_{1}-\zeta_{1} X_{1}+\eta_{1}
$$

Eq.(5) for this system reduces to ( for details, see Ref. [11])

$$
\frac{\partial \rho\left(X_{1}, t\right)}{\partial t}=-\frac{\partial\left(F_{1} \rho\right)}{\partial X_{1}}+D_{1} \frac{\partial^{2} \rho}{\partial X_{1}^{2}}
$$

where the drift term is

$$
F_{1}=-\left(\gamma+2 D_{11}^{\prime}-\nu\right) X_{1}+l
$$

with

$$
l=(2-\nu) \lambda_{11} \sqrt{D_{11}^{\prime} \alpha_{11}}
$$

and

$$
D_{1}=\frac{\left[\alpha_{11} \gamma^{2}+(2-\nu) D_{11}^{\prime} \alpha_{11}\left\{(2-\nu) D_{11}^{\prime}+2 \gamma-2 \gamma \lambda_{11}^{2}-\lambda_{11}^{2}(2-\nu) D_{11}^{\prime}\right\}\right]}{\Gamma^{\prime 2}}
$$

where 


$$
\Gamma^{\prime}=\gamma+2 D_{11}^{\prime}-\nu
$$

Here $D_{11}^{\prime}$ and $\alpha_{11}$ are the multiplicative and additive noise strength respectively. $\lambda_{11}$ is the cross-correlation between the noise processes. $\nu=1$ stands for the Startonovich and $\nu=0$ for the Ito convention, respectively.

The Eq.(37) is very much similar to Eq. (18). Hence, the upper bound for the rate of entropy change can be calculated as in the previous case and the final expression for the upper bound $U_{B}$ is given by

$$
\frac{d S}{d t} \leq U_{B}(t)
$$

where

$$
U_{B}(t)=\frac{\left(2 \beta_{1}^{2} \Gamma^{\prime 2} \sigma_{1}-4 \beta_{1} \Gamma^{\prime} l \sigma_{1}+2 l^{2} \sigma_{1}+\Gamma^{\prime 2} \sigma_{1}^{2}+4 D_{1}^{2}-4 D_{1} \Gamma^{\prime} \sigma_{1}\right)^{1 / / 2}}{\sigma_{1}}
$$

Here the time evolution of $\sigma_{1}(t)$ and $\beta_{1}(t)$ can be written as

$$
\sigma_{1}(t)=\frac{2 D_{1}}{\Gamma^{\prime}}\left(1-\exp \left(-2 \Gamma^{\prime} t\right)\right)+\sigma_{1}(0) \exp \left(-2 \Gamma^{\prime} t\right)
$$

and

$$
\beta_{1}(t)=\frac{l}{\Gamma^{\prime}}\left(1-\exp \left(-2 \Gamma^{\prime} t\right)\right)+\beta_{1}(0) \exp \left(-\Gamma^{\prime} t\right)
$$

The initial conditions for $\sigma_{1}(0)$ and $\beta_{1}(0)$ can be chosen as in Eq.(25). $l, D_{1}$ and $\Gamma^{\prime}$ are determined by Eqs. (39), (40) and (41). Again it is easy to check that for the correlated noise process under stationary condition we obtain the usual equality

$$
\frac{d S}{d t}=0
$$

The time dependence of $U_{B}$ for a correlated noise process (we fix the parameter values as $\gamma=1.0, D_{11}=1.0, \lambda_{11}=0.5, \alpha_{11}=1.0$ and the initial conditions $\left.\sigma_{1}(0)=0, \beta_{1}(0)=0\right)$ is more or less same as that of Fig.1. In Fig.4 we exhibit the variation of $U_{B}$ (at $\left.t=5.0\right)$ with the strength of correlation $\lambda_{11}$. It is interesting to note that although both multiplicative and additive noises are independently and instantaneously correlated their mutual strength 
of correlation $\lambda_{11}$ drives the system away from stationarity more strongly (as compared to the case corresponding to the variation of correlation time $\tau$ in Fig. 2(b)). No minimum, however, is obtained. We mention, in passing, that since the models considered here are linear and are exactly solvable by Green's function of Gaussian form the computed upper bound is an exact one.

\section{CONCLUSIONS}

Based on Fokker-Planck description of color and cross-correlated noise-driven dynamical systems we have shown how the intrinsic properties of a noise process can set an upper bound for the rate of entropy change in a nonequilibrium system. Since the dissipative forces tend to equilibrate the system while an increase in the noise correlation time in a color noise process or an increase in the strength of correlation in cross-correlated noise processes acts in the opposite direction, an interplay of them makes the dynamical system exhibit interesting extremum properties of this upper bound. This is manifested in the

maxima and minima of the bound for the time derivative of Shanon entropy as a function of the strength of dissipation, correlation time or strength of correlation in presence or absence of the external forces acting on the dynamics. Since the color and cross-correlated noise processes occur in many situations in physics and chemistry, the observation made in this paper, we hope, will be useful for understanding the close connection between irreversible thermodynamics and dynamical systems in many related issues.

\section{ACKNOWLEDGMENTS}

The author expresses his deep sense of gratitude to D S Ray for suggesting the present problem and for his kind attention throughout its progress. 


\section{REFERENCES}

[1] B. L. Holian, W. Hoover, and H. Posch, Phys. Rev. Lett. 59, 10 (1987).

[2] N. I. Chernov, G. L. Eyink, J. L. Lebowitz, and Ya. G. Sinai, Commun. Math. Phys. 154, 569 (1993).

[3] D. Ruelle, J. Stat. Phys. 85, 1 (1996).

[4] A. Compte and D. Jou, J. Phys. A 29, 4321 (1996).

[5] P. Gaspard, J. Stat. Phys. 88, 1215 (1997).

[6] D. Brody and B. Meister Phys. Letts. A 204, 93 (1995).

[7] B. Nikolov and B. R. Frieden, Phys. Rev. E 49, 4815 (1994).

[8] B. R. Frieden, Opt. Lett. 14, 199 (1989).

[9] B. C. Bag, J. Ray Chaudhuri, and D. S. Ray, J. Phys. A 33, 8331 (2000).

[10] D. Daems and G. Nicolis, Phys. Rev. E 59, 4000 (1999).

[11] B. C. Bag, S. K Banik and D. S. Ray, Phys. Rev. E 64, 026110 (2001).

[12] B. C. Bag and D. S. Ray, J. Stat. Phys. 96, 271 (1999).

[13] S. Chaudhuri, G. Gangopadhyay, and D. S. Ray, Phys. Rev. E 47, 311 (1993).

[14] K. Lindenberg and B. J. West, The Nonequilibrium Statistical Mechanics of Open and Closed Systems (VCH, New York, 1990).

[15] See, for example, H. Risken, Fokker-Planck Equation (Springer-Verlag, Berlin, 1989).

[16] J. H. Li and Z. Q. Huang, Phys. Rev. E 53, 3315 (1996).

[17] J. H. Li and Z. Q. Huang, Phys. Rev. E 57, 3917 (1998). 


\section{Figure captions}

Fig.1. Plot of upper bound for the time derivative of entropy $\ln U_{B}(t)$ vs time $t$ for the Eq.(31) using $\gamma=1.0, f_{c}=1.0, D^{0}=1, \tau=1.0, \beta(0)=1.0$ and $\sigma(0)=0.0$ (Units are arbitrary).

Fig.2(a). Plot of $U_{B}$ vs correlation time $\tau$ at $t=5.0$ for the Eq.(31) using $f_{c}=0.0$ and values of other parameters same as in Fig.1(Units are arbitrary).

Fig.2(b). Same as in Fig.2(a) but for $f_{c}=1.0$ (Units are arbitrary).

Fig.3(a). Plot of $U_{B}$ vs dissipative constant $\gamma$ at $t=5.0$ for the Eq.(31) using $\tau=1.0$ and values of other parameters same as in Fig.2(a) (Units are arbitrary).

Fig.3(b). Same as in Fig.3(a) but for $f_{c}=1.0$ (Units are arbitrary).

Fig.4. Plot of $U_{B}$ vs noise correlation strength $\lambda_{11}$ at $t=5.0$ for the Eq.(42) using $\gamma=1.0$, $D_{11}=1, \alpha_{11}=1.0, \beta_{1}(0)=1.0$ and $\sigma_{1}(0)=0.0$ (Units are arbitrary). 


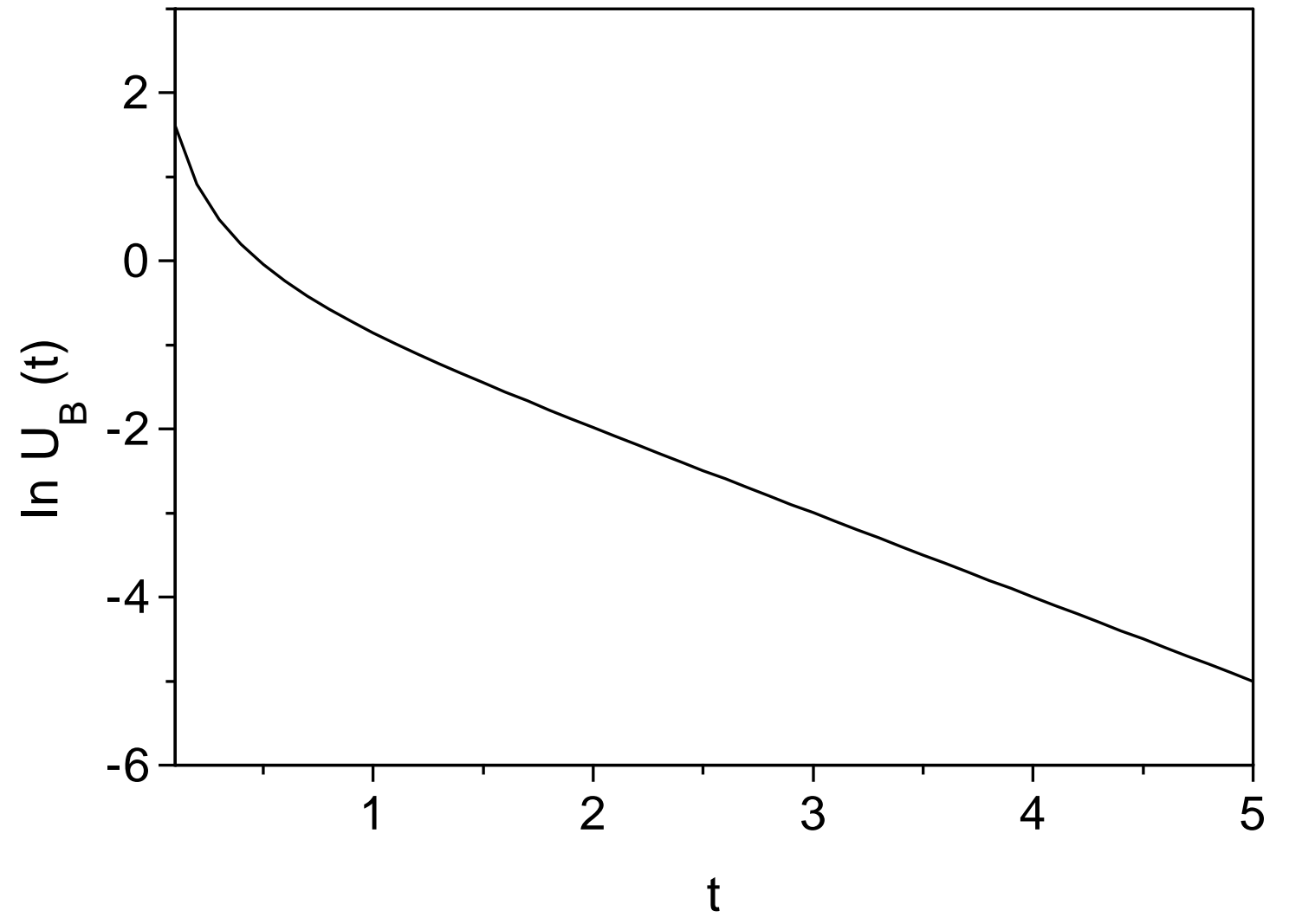

Fig.(1) 


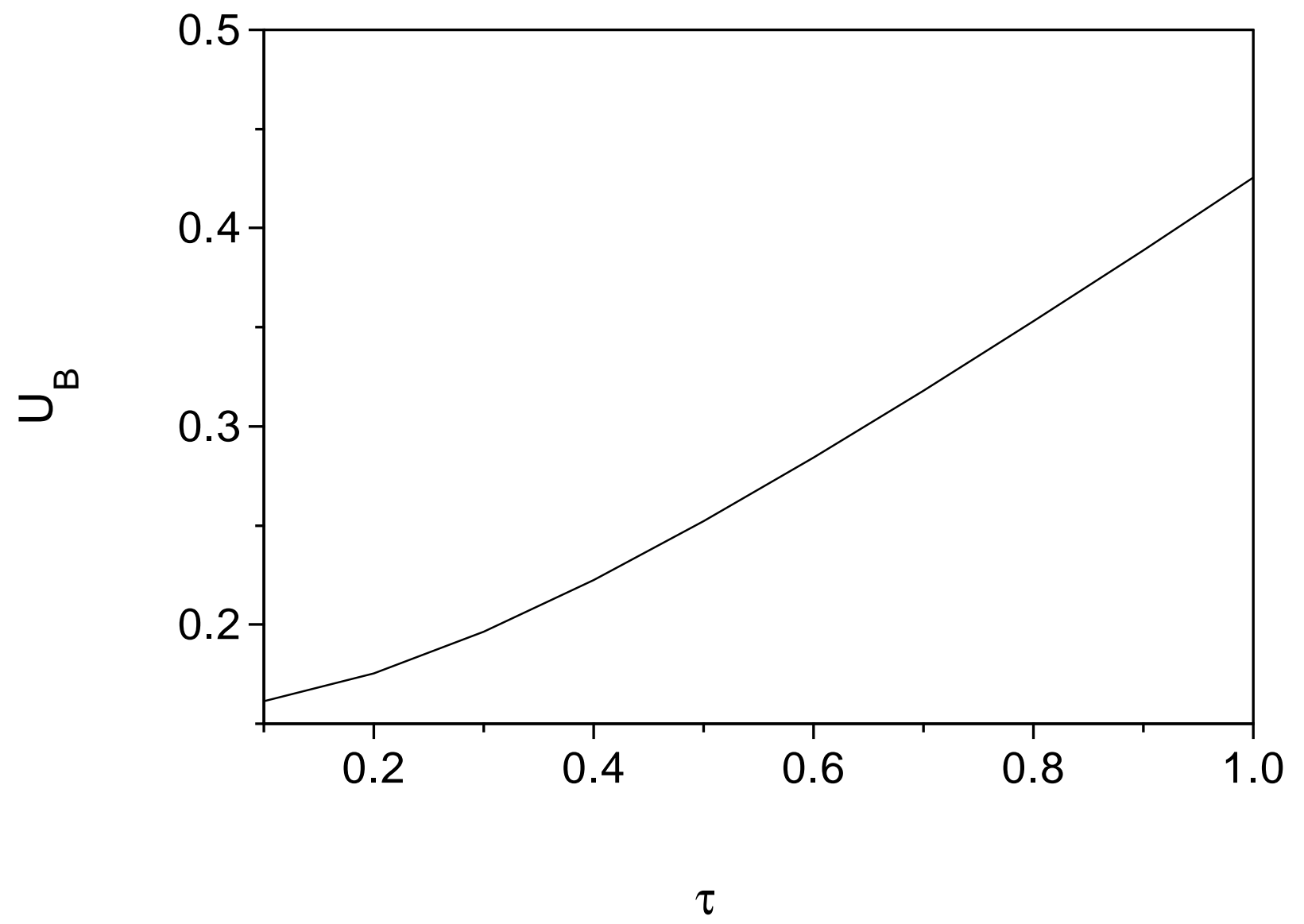

Fig.2(a) 


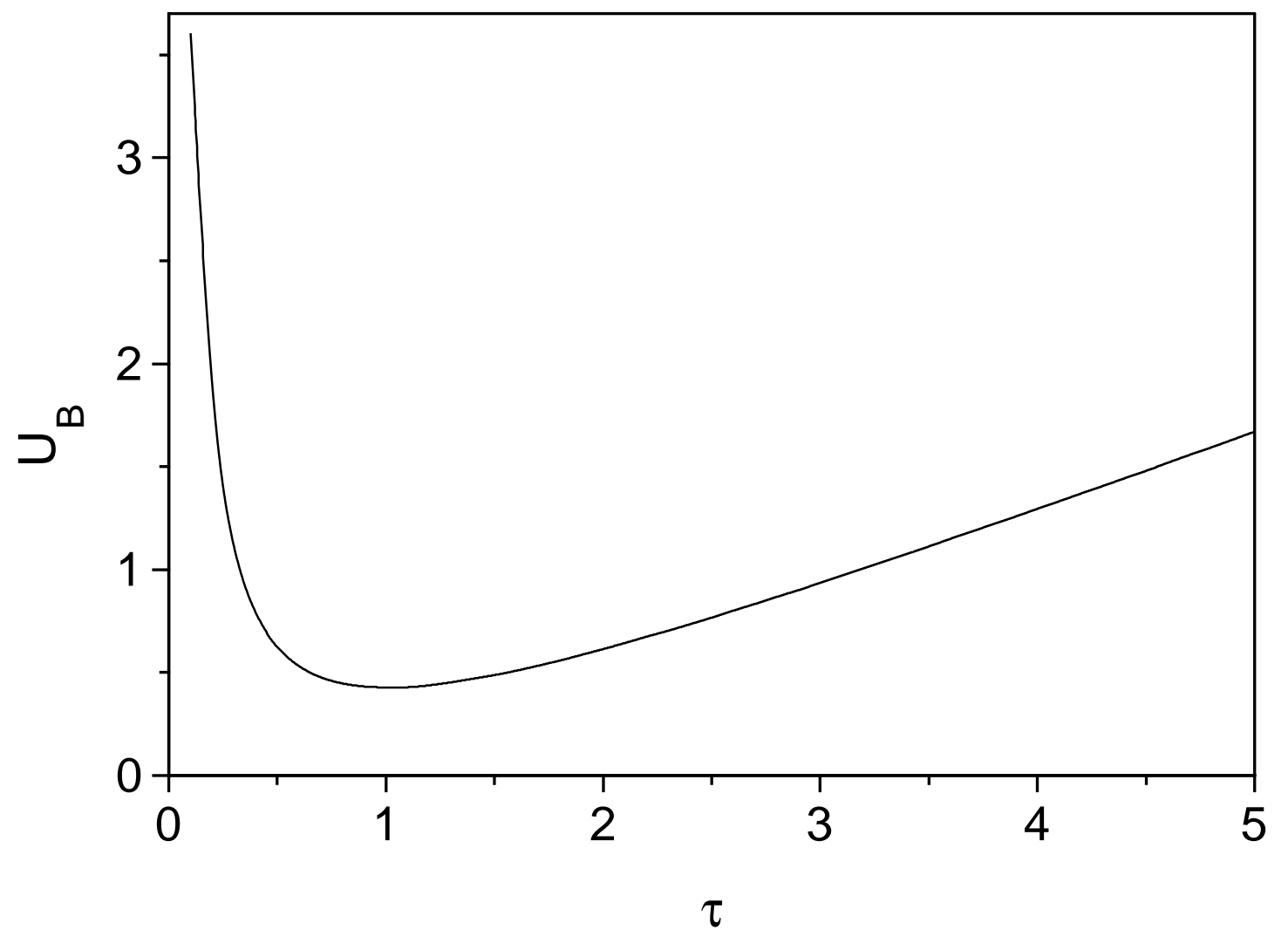

Fig.2(b) 


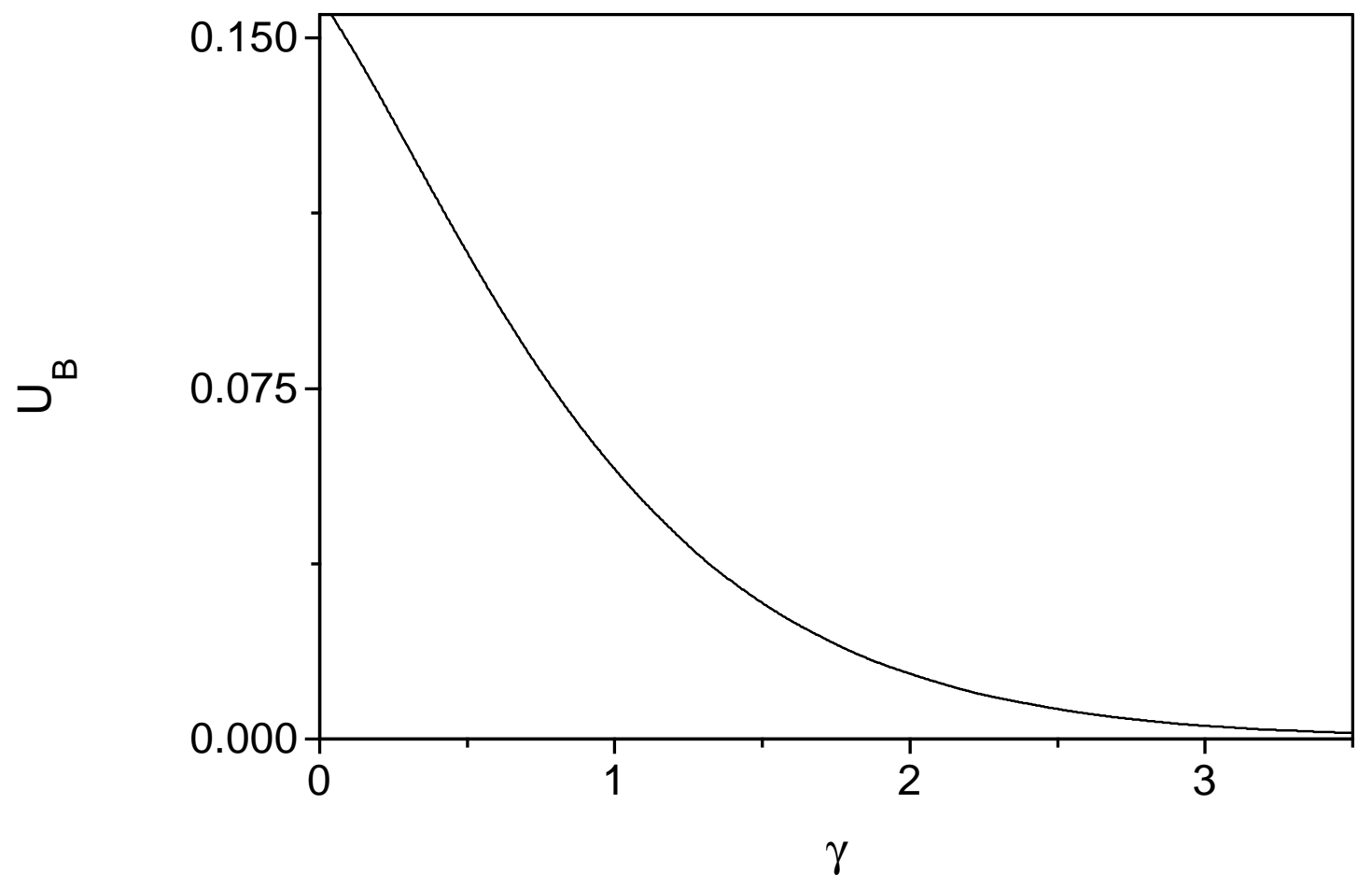

Fig.3(a) 


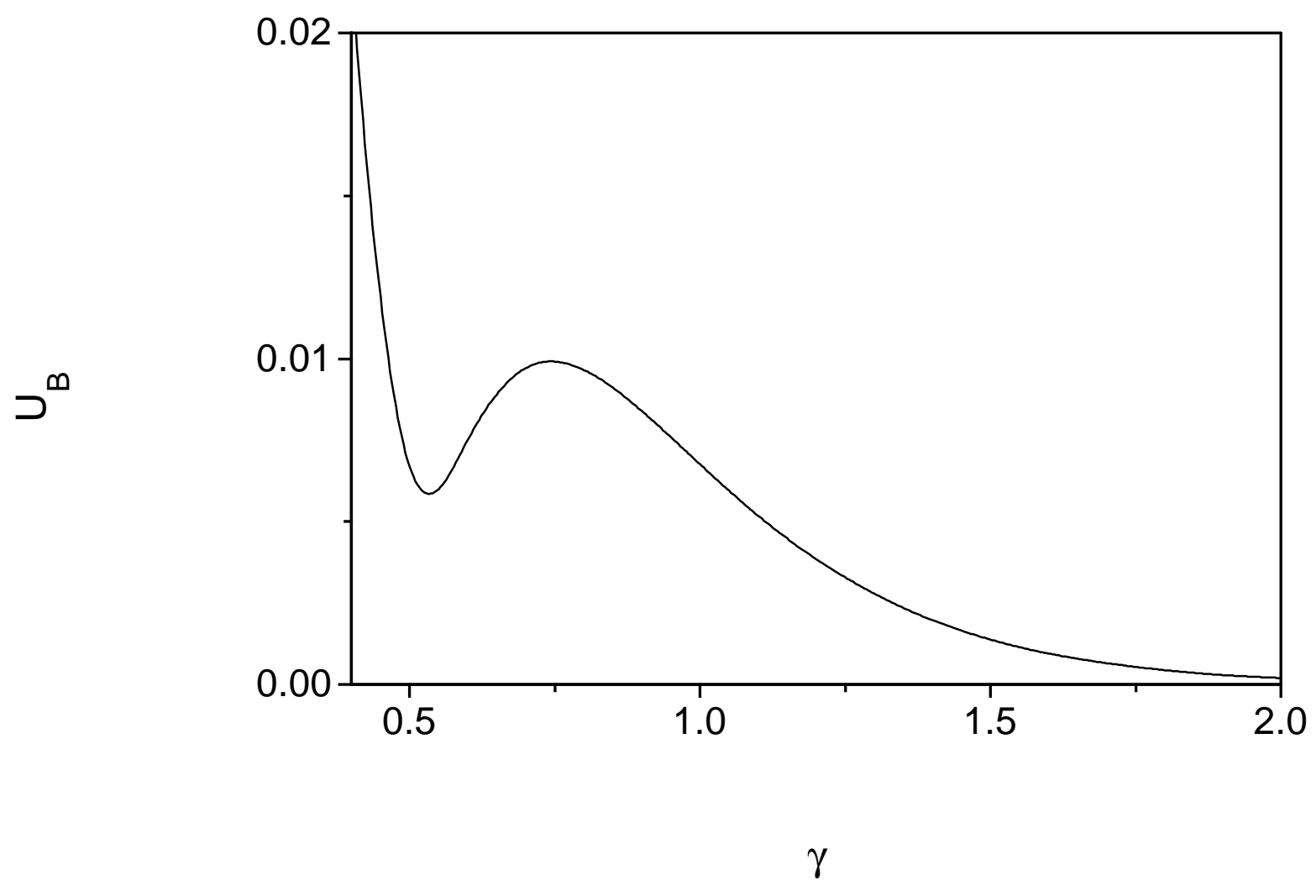

Fig.3(b) 


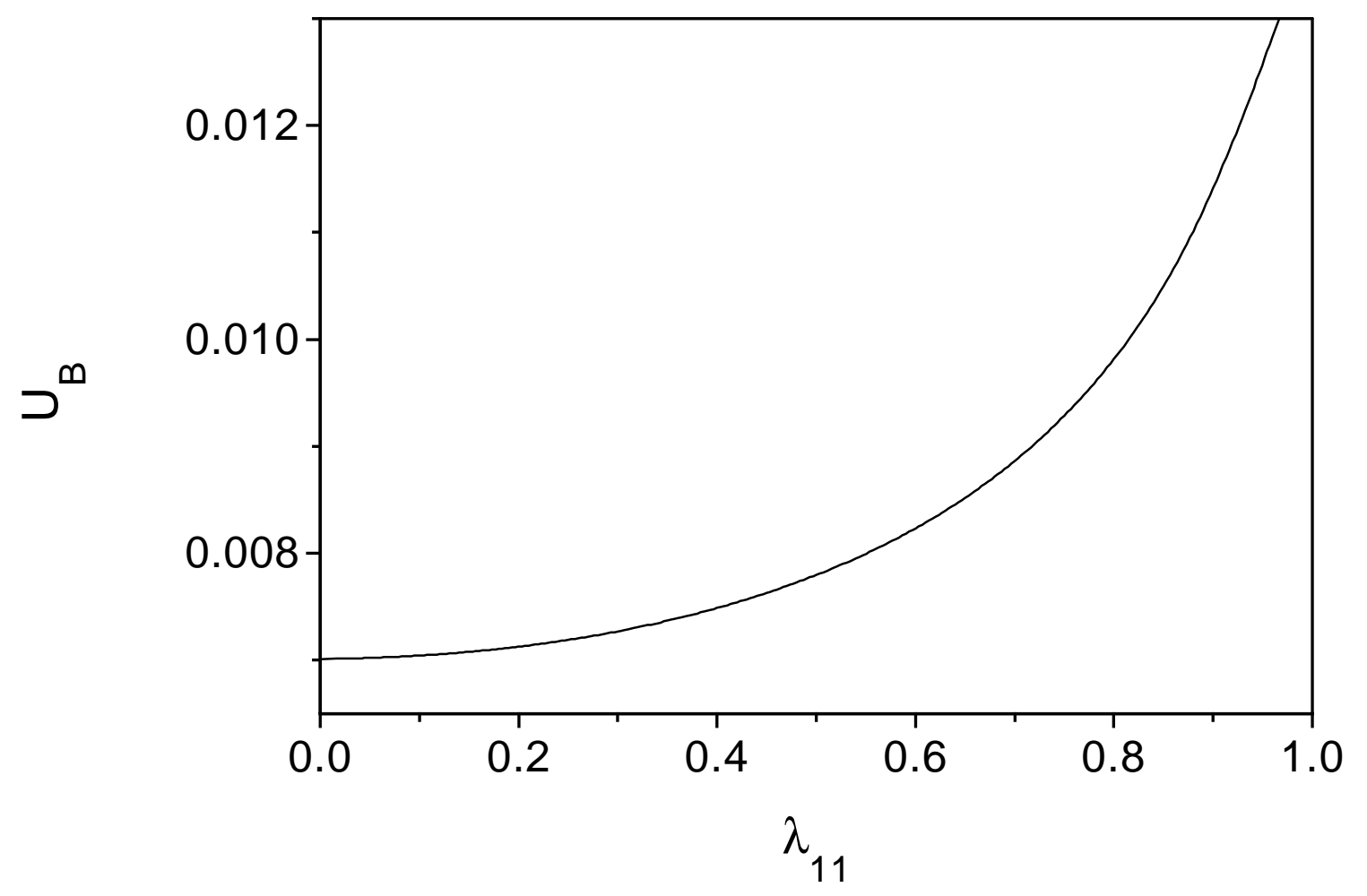

Fig.4 